\title{
DIABETES MELLITUS IN CHILDREN AND YOUNG PEOPLE IN MORECAMBE BAY
}

\author{
Sue Ireland, Consultant \\ Children's Diabetic Services, Westmorland General Hospital
}

The incidence of Type 1 diabetes has increased steadily over the past ten years, particularly in younger children. The national incidence is now two per thousand below sixteen years of age. Morecambe Bay has 160 affected children, with $97 \%$ having Type 1 diabetes and the remainder either MODY, Type 2 or associated with cystic fibrosis.

Type 1 diabetes is an autoimmune disease. Genetic susceptibility depends on several genes with different loci, the strongest linkage being with the HLA antigen on the short arm of chromosome 6. Ninety-five percent of Caucasian type 1 diabetics will carry the HLA DR3 or DR4 antigen. Work in Cambridge is looking at the detailed genetics as part of the human genome project. In Morecambe Bay we are participating in the GRID study (genetic resources investigating diabetes) and approximately half the children in Morecambe Bay are already enrolled in this project.

It is postulated that an environmental trigger precipitates the onset of clinical diabetes in the susceptible individual. Many viruses such as coxsackie B have been implicated and frequently a viral infection will have been present in the weeks before overt symptoms develop. Presentation is more common in the winter when more viruses prevail. It is unclear whether the virus targets the islet B cells specifically or triggers the autoimmune reaction. In addition to viruses other environmental determinants may include certain foods, such as early exposure to cow's milk protein or exposure of the mother to nitrosamine in smoked meat at the time of conception.

As the interaction between diet, insulin, exercise, growth and puberty is a complex one, a multidisciplinary team approach to management is essential, comprising medical, nursing, dietetic, psychological and podiatry input. Morecambe Bay is developing such teams at each of the major hospital sites. We would encourage direct immediate referral to the hospital on the day of diagnosis by the GP as soon as a diagnostic test confirms the child to have glycosuria. It is not necessary for preliminary blood tests to be done prior to referral.

The DCCT (diabetes control and complications trial), which reported in 1993, showed that good control is vitally important to decrease longterm complications and this applies even more to young people than to old. In children this is particularly difficult as children have special needs to grow and develop in physical, intellectual, and psychosocial spheres whilst coping with the sometimes unpredictable and frustrating complexities of diabetes. Tight control in childhood is more likely to result in hypoglycaemic episodes leading to concerns of possible loss of intellectual capacity. As severe hypos are uncomfortable and frightening, this frequently leads to a desire to run sugars on the high side, often in the 10 to 14 range, with consequent poor longterm control. Goals and treatment are summarised in the table below:

\author{
no symptoms or discomfort in everyday life \\ good general health and well-being \\ normal growth and development \\ normal puberty and peer-group relations \\ normal schooling and professional life \\ normal family life including possibility of pregnancy \\ prevention of longterm complications
}

Table: goals of treatment

Pens, devices and insulins are changing and evolving all the time. The traditional regime in the UK, which works well for the majority of children, is a twice-daily injection regime, using pre-mixed insulins, with diet and exercise arranged around the insulin. There is, however, growing evidence that a more flexible approach to insulin administration is associated with a better quality of life, and, from the initial DCCT studies, results in better control. The flexible regime combines a long-acting insulin to provide background levels together with one of the rapidly absorbed insulin analogues given several times a day before each carbohydrate intake. This system is also known as a basal bolus system. It involves carbohydrate counting and the adjustment of the rapid-acting insulin to sugar levels, activity and anticipated intake. We have already used this successfully in children as young as nine and anticipate its use in even younger children. There is, however, no doubt that it requires hard work on the part of the patient!

Continuous insulin infusion pumps allow even greater flexibility with the variation in basal requirements of insulin but at present is not being used here. We expect this to change fairly soon as a number of young people satisfy the NICE guidelines and are keen for the challenge.

The currently used analogue insulins include Humalog and NovoRapid which are modified to be absorbed more quickly, whereas Insulin Glargine and Detemir have a relatively constant absorption over a longer period of time.

Even children on fixed insulin regimes should be doing four to five or more blood sugar tests per day, so there is a greater need for access to blood sugar test strips.

\section{WHERE ARE WE GOING WITH PAEDIATRIC DIABETES IN MORECAMBE BAY?}

Education of the patient for self-management in the long term is a vital part of good diabetic control. We are currently looking at more formal educational programmes. The diabetes nurse specialists provide a lot of input to schools to help in maintaining healthy happy childhoods for affected 
children within the school environment. At the larger senior schools the diabetes nurse specialists run drop-in sessions. The youngsters themselves set the agenda.

We have also developed a paediatric diabetes group for professionals across the bay, which meets regularly to update and evolve protocols for acute management at home, school and hospital. The service is clearly aiming to meet NSF guidelines for the management of diabetes and we have a representative on the local implementation team for this. We have also been involved in the development of the NICE guidelines for the management of paediatric diabetes with one of our DSN's on the committee and one of the doctors in the consultation group. We have participated in the diabetes UK audit for the last two years and we are now a pilot site for the new National Diabetes Data Set Project. We are committed to audit and will soon be doing a project to look at patient satisfaction and the requirements from the diabetes team.

There are some aspects of the service which clearly still need a considerable amount of work. We are trying to target these by the involvement of the Child Health Executive. Urgent specific needs include increased dietetic support in the Barrow and South Lakes areas and easy access to counselling or psychology input. Twenty percent of young people with diabetes will have a serious psychological problem which will contribute towards poor diabetic control.
As a group the diabetes team welcomes comments or suggestions from members of the PCT and would like to think we are approachable should there be any worries or queries. We are aware, for example, of the need to be a little more exact when requesting insulin for our patients as there is a bewildering array of possibilities and of course GPs need clear guidance as to what we require!

\section{LEARNING POINTS}

- The incidence of Type I diabetes continues to increase steadily in young people

- Any new cases picked up in the community should be referred directly to the Paediatric Ward, RLI, 01524 583467/68 (for Lancaster and Kendal) or FGH 01229491090 (for Barrow). There is no need for prior blood tests in the community.

- Good control correlates with better longterm outcome and fewer complications

- Newer treatment methods include flexible insulin regimes, using a background of longlasting insulin with variable dose boluses of a short-acting insulin

- Formal education programmes involving the child, family, school and GP are being developed

- Increased dietetic support is urgently required, particularly for the Barrow and South Lakes areas

- $20 \%$ of diabetic children will have psychological problems. Increased counselling services are urgently needed for the whole of Morecambe Bay

\section{Undergraduate MBMJ Prize}

The Leese Bequest has agreed to contribute to a prize of $£ 200$ for the best submission written by an undergraduate undertaking work in the fields of respiratory or cardiovascular medicine. This could be a case report, results of an audit or original research. The successful piece of work will be published in the MBMJ and should be between 2500 and 3000 words long.

Entries will be judged at the end of the academic year (31 August 2005) and anyone interested in participating is encouraged to speak to Dr A Brodison, Consultant Physician at RLI. 